\title{
Pembrolizumab in MSI-high Pancreatic Sarcomatoid Carcinoma
}

\author{
Mayrhofer K* \\ Department of Medical Oncology, Klinik Ottakring, \\ Austria \\ *Corresponding author: Mayrhofer K, Department of \\ Medical Oncology, Klinik Ottakring, Montleartstraße 37, \\ 1160 Vienna, Austria
}

Received: January 03, 2021; Accepted: February 03, 2021; Published: February 10, 2021

\begin{abstract}
We report the outcome of a patient with MSI-high metastatic pancreatic sarcomatoid carcinoma refractory to multiple lines of chemotherapy treated successfully with pembrolizumab.

In November 2015, our patient presented with epigastric pain leading to radiologic workup. A lesion in the pancreas as well as liver metastasis were diagnosed; liver biopsy revealed a poorly differentiated sarcomatoid carcinoma. After having received every approved chemotherapy for pancreatic cancer his disease eventually progressed so that we reached out for novel therapeutic strategies. Because of the tumor being MSI high, treatment with pembrolizumab was commenced in May 2017. Clinical response with better overall quality of life was soon reported and repeated CT scans showed an ongoing partial response leading to a near complete remission in the latest scan obtained. Adverse events during the course of therapy included immune mediated arthralgia grade 1 , colitis grade 2 and pneumonitis grade 1 which were managed by administration of glucocorticoids without interruption of immunotherapy. To our knowledge, this is the first case of a patient with MSI high metastatic sarcomatoid carcinoma of the pancreas successfully treated with immunotherapy for more than three years.
\end{abstract}

Keywords: Pembrolizumab; Sarcomatoid carcinoma; Pancreatic cancer

\section{Case Presentation}

Because of epigastric pain our patient underwent abdominal ultrasound which revealed hepatic lesions as well as a tumor in the pancreas. The patient was then referred to our clinic where a Computed Tomography (CT) and Magnetic Resonance Imaging (MRI) was performed, confirming the sonographic findings. Liver biopsy showed metastasis of a poorly differentiated Sarcomatoid Carcinoma (SC). Immunohistochemistry was unable to definitely attribute the tissue sample to a specific organ. The radiologic workup however indicated that the metastasis originated from the pancreas because of pathologic locoregional lymph nodes and a high correlation between the hepatic lesions and the pancreatic tumor in terms of radiographic characteristics. Hence, the diagnosis of a metastatic sarcomatoid pancreatic cancer was made and discussed with the patient. He presented in a good performance status, comorbidities were a well-controlled arterial hypertension and insomnia requiring oral medication. Family history was positive with his father having had pancreatic cancer, his sister breast cancer and his daughter colon cancer. With the patient's consent, a therapy consisting of gemcitabine and albumin-bound paclitaxel was initiated in November 2015. Tolerance to therapy was very good and after 6 cycles, staging CT showed a complete remission of the malignant liver lesions and a partial remission of the primary tumor. MRI and contrast enhanced ultrasound confirmed complete disappearance of all liver metastasis. Based on these results, our tumor board recommended to pause chemotherapy, do a follow up imaging 8 weeks later and if there are no signs of disease progression, proceed to surgery of the primary tumor. Follow up imaging with CT and MRI excluded progressive disease and the patient underwent whipple resection of the pancreatic tumor. Histology revealed stage ypT3 N1 V1 L0 with necrosis of approximately $50 \%$ of the tumor tissue. The patient recovered quite well without major complications from surgery.

Due to the extraordinary response to first line chemotherapy, the decision to continue therapy postoperatively was made. After receiving another two cycles of gemcitabine and albumin-bound paclitaxel, however, disease progression in the form of appearance of new hepatic metastasis as well as retroperitoneal lymphadenopathy was evident in October 2016.

Second line chemotherapy according to the FOLFOX (folinic acid, fluorouracil, oxaliplatin) protocol was commenced in the same month. Therapy tolerance was poor with prolonged nausea and worsening performance status during the course of therapy. After the fourth application, the patient was admitted to our ward due to prolonged diarrhea, vomiting and grade 4 neutropenia. He was able to continue therapy with a reduced dose $(75 \%)$ of fluorouracil afterwards. The first staging CT after 9 applications of FOLFOX showed mixed response of the liver lesions. Therapy was then switched to nanoliposomal irinotecan combined with fluorouracil in March 2017. Treatment tolerance was poor mainly caused by fatigue symptoms. Therapy had to be interrupted due to an infectious complication after two applications. Moreover, signs of clinical progression led to early restaging by $\mathrm{CT}$, which confirmed progressive disease of the liver (Figure 1). The patient was now refractory to third line therapy and also unable to tolerate any further chemotherapy. In this situation, we reached out for novel therapeutic strategies. The tissue sample of the whipple resection was analysed for Microsatellite Instability (MSI) 


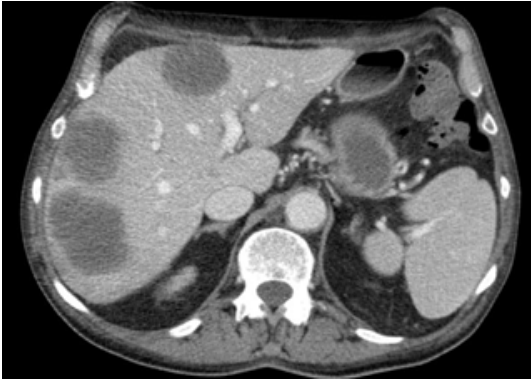

Figure 1: CT scan in May 2017 showing multiple liver metastasis.

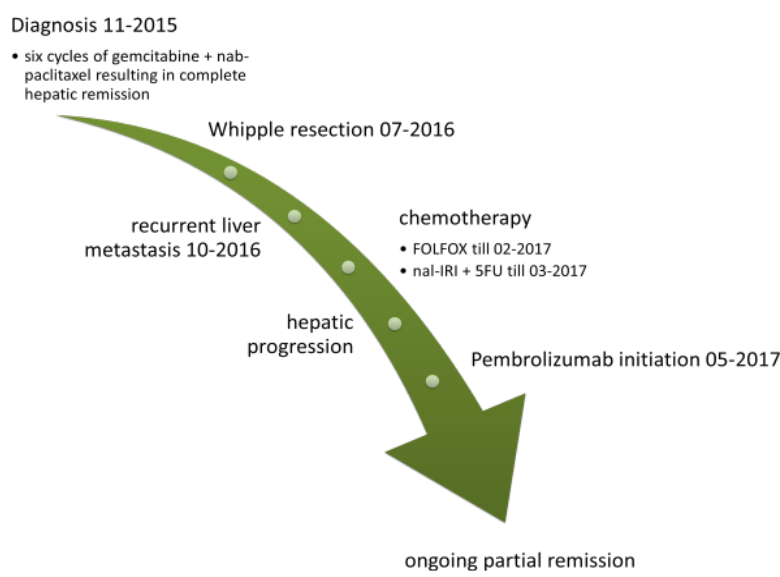

Figure 2: Diagnostic and therapeutic timeline.

by immunohistochemistry and turned out to be MSI high. Given the extraordinary pivotal data of pembrolizumab in deficient Mismatch Repair (dMMR) gastrointestinal tumors [1-8], pembrolizumab was started after positive recommendation of our tumor board in May 2017. Following treatment initiation, the patient soon reported a better overall quality of life and less fatigue. Repeated CT scans showed an ongoing partial response and overall treatment tolerance was well. In November 2017 he developed mild generalised arthralgia probably immunotherapy associated with a prompt resolution by administration of intermediate dose glucocorticoids. Therapy hasn't had to be interrupted and glucocorticoid dose was tapered to $7.25 \mathrm{mg}$ prednisolone daily. The patient achieved a long-lasting response to pembrolizumab therapy. In the further course of his disease, he was admitted to our ward in June 2019 due to pneumonia and immunotherapy associated colitis. CT scan confirmed pneumonia and also revealed mild (grade 1) signs of pneumonitis. The SC still was in near complete remission with only two small liver lesions remaining. With appropriate management consisting of antibiotics and glucocorticoids he recovered quickly and was discharged after one week. Corticosteroids were tapered to a daily dose of $1 \mathrm{mg}$ dexamethasone. Pembrolizumab therapy was continued afterwards and to date (December 2020) is still ongoing (Figure 2). The latest obtained CT scan in August 2020 showed a near complete remission of the liver metastasis (Figure 3).

\section{Discussion}

\section{Sarcomatoid carcinoma of the pancreas}

According to the 2010 world health organisation histologic

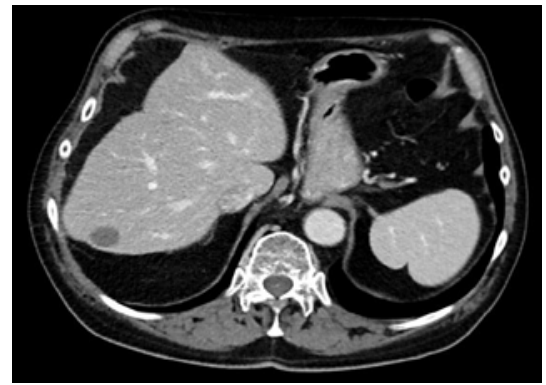

Figure 3: CT scan in August 2020 showing long lasting response.

classification of pancreatic tumors, sarcomatoid carcinoma belongs to the group of undifferentiated carcinomas [1]. It is an extremely rare and highly aggressive subtype with only a few cases having been reported in the literature [2,3]. Reported outcomes are poor with survival not exceeding more than several months. This is in sharp contrast to our patient who is alive 4 years after initial diagnosis. This is mainly caused by his excellent response to immunotherapy and also to the initial complete disappearance of hepatic metastasis to front line chemotherapy. Little is known about optimal therapy of sarcomatoid carcinomas in general and for pancreatic origin in particular. We treated the patient with chemotherapy protocols analogue to pancreatic adenocarcinoma and he achieved an extraordinary response to first line gemcitabine and albumin bound paclitaxel. However, response didn't last long and further line chemotherapy showed no activity.

\section{Immunotherapy in renal and lung sarcomatoid carcinoma}

Immunotherapy represents a new therapeutic strategy in patients with SC. In sarcomatoid renal cell carcinoma, subgroup analyses of the CheckMate214 trial revealed an ORR of 57\%, a CR rate of $18 \%$ and a median overall survival of 31 months [4]. Nivolumab and ipilimumab therefore is recommended as first line therapy in intermediate and poor risk renal SC. Data from a retrospective evaluation of treatment outcomes of 39 patients with SC of the lung also shows clinical activity of single agent immunotherapy with an ORR of $38.5 \%$, median PFS of 4.59 months and median OS of 20 months [5]. However, immunotherapy is well established in adenocarcinoma / squamous cell carcinoma of the lung and clear cell carcinoma of the kidneys, raising doubt whether this positive data in renal and lung SC can be replicated in SC of other origin. Further research is necessary to define the role of immunotherapy on sarcomatoid carcinomas of gastrointestinal origin. The long lasting response achieved in our patient is not attributed to the histologic subtype of SC but rather to the tumor being MSI high.

\section{PD-L1 Blockade in pancreatic adenocarcinoma}

Despite its advance in the treatment of various tumor types, Programmed Death-Ligand 1 (PD-L1) inhibition provided dismal results in unselected patients with pancreatic cancer [6]. This is attributed to an immunosuppressive tumor microenvironment as well as the low immunogenicity of pancreatic tumors.

To better select patients who are likely to benefit from checkpoint inhibition, several biomarkers have been investigated. PD-L1 on tumor cells and tumor infiltrating immune cells, respectively, is used as a predictive marker in a variety of tumor types including lung, 
breast, urothelial, head and neck and gastric cancer. In pancreatic cancer, however, PD-L1 doesn't exhibit any predictive value $[6,7]$.

A dMMR system causes a high amount of Deoxyribonucleic Acid (DNA) replication errors leading to lots of somatic mutations and MSI. This results in a high load of neoantigens on the surface of tumor cells which leads to an increased immunogenicity that can be further enhanced by the use of immunotherapy.

Several large multicenter single arm basket trials (Keynote 12 , $16,28,158,164)$ evaluate the role of pembrolizumab in MSI-high and dMMR solid tumors. Due to the overwhelming primary results of combined data of these trials, the food and drug administration approved pembrolizumab in MSI-high or dMMR gastrointestinal solid tumors [8]. Most of the 149 patients included in the primary analysis had colon cancer while only 6 patients had pancreatic cancer. Of these, 5 were reported having achieved a partial remission. The results of the keynote 16 trial have been published in 2017 with an objective response rate of $53 \%$ and median PFS and OS not reached after a median follow up of 12.5 months [9]. A total of 8 patients with pancreatic cancer were included. There were 2 complete remissions, 3 partial remissions, one patient with stable disease and 2 patients experienced clinical progression before the first staging computed tomography. Despite the low number of patients with pancreatic cancer in these trials, concordant results between the different tumor types makes pembrolizumab a viable treatment option in all solid tumors exhibiting dMMR including pancreatic cancer. Major guidelines like ASCO and NCCN recommend testing for dMMR by immunohistochemistry, polymerase chain reaction or next-generation sequencing and if positive, pembrolizumab is recommended as second or later line therapy, respectively $[10,11]$.

\section{Conclusion}

To our knowledge, this is the first case of a MSI high metastatic sarcomatoid carcinoma of the pancreas treated with immunotherapy. Being refractory to third line chemotherapy, our patient achieved a near complete remission to pembrolizumab therapy with an ongoing response since more than three years. This case report confirms the important role of immune checkpoint inhibition in dMMR tumors and discusses its role in sarcomatoid carcinoma and pancreatic cancer.

\section{References}

1. Bosman FT. World Health Organization, International Agency for Research on Cancer, editors. WHO classification of tumours of the digestive system. $4^{\text {th }}$ ed. Lyon: International Agency for Research on Cancer. 2010; 417.

2. Ren $C L$, Jin $P$, Han CX, Xiao Q, Wang DR, Shi L, et al. Unusual early-stage pancreatic sarcomatoid carcinoma. World J Gastroenterol WJG. 2013; 19: 7820-7824.

3. Yepuri N, Pruekprasert N, Naous R. High-grade malignant pancreatic neoplasm with sarcomatoid features. AME Case Rep. 2018; 2: 39.

4. Motzer RJ, Tannir NM, McDermott DF, Arén Frontera O, Melichar B, Choueir TK, et al. Nivolumab plus Ipilimumab versus Sunitinib in Advanced Renal-Cell Carcinoma. N Engl J Med. 2018; 378: 1277-1290.

5. Charlotte D. Efficacy of immune checkpoint inhibitors in lung sarcomatoid carcinoma: data from a French multicentric cohort. ESMO 2018 Congress.

6. Brahmer JR, Tykodi SS, Chow LQM, Hwu WJ, Topalian SL, Hwu P, et al. Safety and Activity of Anti-PD-L1 Antibody in Patients with Advanced Cancer. N Engl J Med. 2012; 366: 2455-2465.

7. Feng M, Xiong G, Cao Z, Yang G, Zheng S, Song X, et al. PD-1/PD-L1 and immunotherapy for pancreatic cancer. Cancer Lett. 2017; 407: 57-65.

8. Lemery S, Keegan P, Pazdur R. First FDA Approval Agnostic of Cancer Site - When a Biomarker Defines the Indication. N Engl J Med. 2017; 377 : 1409-1412.

9. Le DT, Durham JN, Smith KN, Wang H, Bartlett BR, Aulakh LK, et al Mismatch repair deficiency predicts response of solid tumors to PD-1 blockade. Science. 2017; 357: 409-413.

10. Sohal DPS, Kennedy EB, Khorana A, Copur MS, Crane CH, Garrido-Laguna I, et al. Metastatic Pancreatic Cancer: ASCO Clinical Practice Guideline Update. J Clin Oncol. 2018; 36: 2545-2556.

11. Tempero M, Malafa M. National Comprehensive Cancer Network Clinica Practice Guideline Pancreatic Adenocarcinoma V1. 2020; 2019. 